\title{
Quantitative assessment of acute kidney injury by noninvasive arterial spin labeling perfusion MRI: a pilot study
}

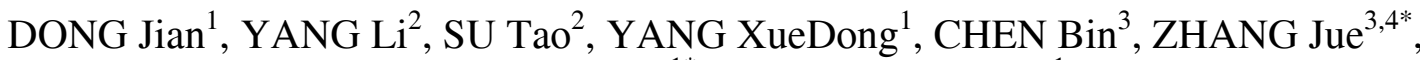 \\ WANG XiaoYing $^{1 *} \&$ JIANG XueXiang ${ }^{1}$ \\ ${ }^{1}$ Department of Radiology, Peking University First Hospital, Beijing 100034, China; \\ ${ }^{2}$ Department of Nephrology, Peking University First Hospital, Beijing 100034, China; \\ ${ }^{3}$ Academy for Advanced Interdisciplinary Studies, Peking University, Beijing 100871, China; \\ ${ }^{4}$ College of Engineering, Peking University, Beijing 100871, China
}

Received January 22, 2013; accepted April 24, 2013; published online June 3, 2013

\begin{abstract}
The kidneys are essential for maintaining homeostasis, are responsible for the reabsorption of water, glucose and amino acids, and filter the blood by removing waste. Acute kidney injury (AKI) is a syndrome characterized by the rapid loss of renal excretory function and the accumulation of end metabolic products of urea and creatinine. AKI is associated with the later development of chronic kidney disease and end-stage kidney disease, and may eventually be fatal. Early diagnosis of AKI and assessments of the effects of treatment, however, are challenging. The pathophysiological mechanism of AKI is thought to be the imbalance between oxygen supply and demand in the kidneys. We have assessed the ability of arterial spin labeling (ASL) perfusion magnetic resonance imaging (MRI), without the administration of contrast media, to quantify renal blood flow (RBF) non-invasively. We found that RBF was significantly lower in AKI patients than in healthy volunteers. These results suggest that ASL perfusion MRI, a noninvasive measurement of RBF, may be useful in the early diagnosis of AKI.
\end{abstract}

arterial spin labeling (ASL), acute kidney injury (AKI), renal blood flow (RBF), magnetic resonance imaging (MRI)

Citation: Dong J, Yang L, Su T, et al. Quantitative assessment of acute kidney injury by noninvasive arterial spin labeling perfusion MRI: a pilot study. Sci China Life Sci, 2013, 56: 745-750, doi: 10.1007/s11427-013-4503-3

Acute kidney injury (AKI) is a frequently observed condition linked with the later development of chronic kidney disease, end-stage kidney disease, and mortality. AKI has been reported to affect more than 5000 per million people not on dialysis therapy and 295 per million on dialysis therapy per year [1-3]. The advent of the RIFLE (risk, injury, failure, loss, end-stage kidney disease) and Acute Kidney Injury Network (AKIN) criteria has improved the diagnosis of AKI [4], and decreased renal blood flow (RBF), which causes renal ischemia, has been proposed as central to the pathogenesis of septic acute renal failure [5]. These criteria, however, have been based on serum creatinine

*Corresponding author (email: cjr.wangxiaoying@vip.163.com; zhangjue@pku.edu.cn) concentration and urine output, both of which have relatively poor specificity and sensitivity during the early period of AKI [6-9]. At present, therefore, there are no effective biomarkers for AKI, making early diagnosis of AKI and the assessment of the effects of therapy difficult [10].

Noninvasive functional magnetic resonance imaging (MRI) provides a variety of effective methods to evaluate renal function in vivo without administration of contrast media. The arterial spin labeling (ASL) perfusion technique, in which inflowing blood is labeled magnetically, can be used in the absolute quantification of renal blood flow $(\mathrm{RBF})$, especially in patients with impaired renal function [11-15]. ASL was found to be better able to quantify cortical RBF than dynamic contrast enhanced MRI [16]. The 
reproducibility of the flow-sensitive alternating inversion recovery (FAIR) ASL perfusion technique over a broad range of renal function has been demonstrated in the cortices of native and transplanted kidneys [17]. Moreover, by measuring kidney perfusion, ASL demonstrated a potential capability for assessing renal disease in both native and transplanted kidneys $[12,17,18]$. To our knowledge, however, quantitative ASL analysis has not been used in the diagnosis of AKI. We therefore performed a pilot study to investigate the feasibility of noninvasive ASL perfusion MRI in the diagnosis of AKI.

\section{Materials and methods}

\subsection{Subjects}

From October 1, 2011 to December 1, 2012, 13 AKI patients and 18 healthy volunteers were recruited. Patients were included if they (a) were adults diagnosed with AKI by referring nephrologists, (b) were scheduled to undergo percutaneous renal biopsy three days after MR examination and prior to therapy, (c) had no signs of other kidney diseases, such as renal artery stenosis and urolithiasis, as shown by ultrasonography in our hospital while being in patients, and (d) had normal cardiac function. Healthy volunteers were included if they (a) were adults with no history of renal or cardiac diseases, and (b) had normal serum creatinine concentrations one week before MR scanning.

Patients underwent MR examinations before initial of therapy. All subjects refrained from food and fluids for $6 \mathrm{~h}$ and were trained in breath-holding techniques training before MR examination. Serum creatinine concentrations were measured in all subjects before MR examination.

This prospective study was compliant with the Health Insurance Portability and Accountability Act (HIPAA) and was approved by our institutional review board. All subjects provided written informed consent and were compatible with MR scanning.

\subsection{MR protocols}

All examinations were performed on a 3.0T MR scanner (Signa Excite; GE Healthcare, Milwaukee, WI) using a commercial Torso PA coil (GE Medical Systems). The scout images were scanned with a gradient echo sequence in three oblique planes through the center of each kidney. Oblique-coronal T2-weighted imaging through the center of the kidney was performed for anatomical evaluation using a single shot fast spin echo (SSFSE) sequence with the parameters: $T R=1100 \mathrm{~ms}, T E=60 \mathrm{~ms}$, slice thickness $=5 \mathrm{~mm}, 1 \mathrm{~mm}$ interval, matrix $192 \times 256$, flip angle $130^{\circ}$, bandwidth $=$ $62 \mathrm{kHz}$, FOV 35-40 cm.

ASL images were acquired for quantitative measurements of RBF by applying the fluid alternating inversion recovery (FAIR) sequence with the combination of a sin- gle-shot fast spin-echo (SSFSE) imaging (FAIR-SSFSE). One single oblique coronal slice of ASL was scanned in the same position through the center of the kidney with the parameters: field of view $(\mathrm{FOV})=40 \mathrm{~cm}$, matrix $=128 \times 128$, slice thickness $=5 \mathrm{~mm}, T R=3500 \mathrm{~ms}, T E=60 \mathrm{~ms}$, flip angle $=$ $90^{\circ}$, bandwidth $=62.5 \mathrm{kHz}$. The slab thickness was set at 20 $\mathrm{mm}$ for section selective inversion in the FAIR preparation, to avoid artifacts from incompletely inverted spins at the margins of the readout slice, with no feeding vessels included.

At the beginning of the scanning process, inversion radiofrequency pulses were used to label the protons in the arterial blood feeding both kidneys, with the magnetically tagged blood acting as an endogenous tracer. All six ASL images were scanned at the same slice, including three tag images with section selective inversion and three control images with global inversion. The inversion time $(T I)$ between the tag and control images was fixed at $1400 \mathrm{~ms}$ to ensure the tagged protons reached the capillary bed and exchanged with protons in the surrounding tissue; the tag image was then subtracted from the control image to remove signals of static tissue, yielding residual signals for quantification of RBF. $M_{0}$ images were acquired using the SSFSE sequence, with the same parameters as the prior FAIR-SSFSE sequence without FAIR preparation, and the repetition time $(T R)$ was set at $6 \mathrm{~s}$ to allow for the complete recovery of blood signals between measurements.

All participants were instructed to hold their breath for 20-30 s during each acquisition, with ASL imaging performed twice. The total acquisition time for ASL imaging was limited to $1 \mathrm{~min}$.

\subsection{Data processing}

A quantitative model for calculating RBF from FAIR techniques, using extended Block equations [19], was used to simulate $\Delta M(T I)$, the difference in longitudinal magnetization between FAIR images with section-selective preparation and those with global preparation:

$$
\Delta M(T I)=M_{\text {sel }}(T I)-M_{\text {nonsel }}(T I)=2 M_{0} T I \frac{f}{\lambda} \mathrm{e}^{T I / T_{1}},
$$

where $\Delta M$ is the difference in magnetization between section-selective $\left(M_{\text {sel }}\right)$ and nonselective $\left(M_{\text {nonsel }}\right)$ measurements; $M_{0}$ represents the tissue equilibrium magnetization per unit mass of tissue; $T_{1}$ is the longitudinal relaxation time of tissue; $f$ is the perfusion rate (usually expressed in milliliters per $100 \mathrm{~g}$ per minute); and $\lambda$ is the blood-tissue water partition coefficient, which is thought to be nearly constant at 0.80. Perfusion maps can be calculated pixel-by-pixel by analyzing $\Delta M$ at a given $T I, M_{0}$ and the $T_{1}$ using the equation:

$$
f=\frac{\lambda \cdot \Delta M(T I) \cdot f}{2 \cdot T I \cdot M_{0}} \mathrm{e}^{T I / T_{1}} .
$$

All ASL images were post-processed in Matlab software 
(MathWorks Inc. Natick, MA). After correction for motion artifacts, the final $\Delta M$ images were generated by averaging and subtracting section-selective and global inversion images acquired in two sets. The $T_{1}$ values for the renal cortex and medulla were set at 1142 and $1545 \mathrm{~ms}$, respectively [20, 21]. Pixels with perfusion values higher than $600 \mathrm{~mL} \mathrm{~min}^{-1}$ $100 \mathrm{~g}^{-1}$ in the cortex and higher than $250 \mathrm{~mL} \mathrm{~min}^{-1} 100 \mathrm{~g}^{-1}$ in the medulla were excluded from the RBF evaluation. Multiple regions of interest (ROIs) with areas of 50-100 $\mathrm{mm}^{2}$ in the perfusion map, were drawn in the cortex and medulla of both kidneys (Figure 1) [16], and RBFs in the cortex and medulla were separately calculated by averaging all ROI data in these areas. The measurements were performed on both ASL images for each subject, and the averaged value was used as the final RBF.

\subsection{Statistical analysis}

All statistical analyses were performed using SPSS 16.0 (SPSS Inc., Chicago, IL). Quantitative data are presented as mean \pm standard deviation (SD). The RBFs of the cortical and medullary kidney were compared using independent $t$ tests. The correlations between serum creatinine concentrations and the RBFs of cortical and medullary kidney were determined. A $P$-value less than 0.05 was defined as statistically significant.

\section{Results}

MR examinations were performed in 13 patients (eight males and five females, ranging in age from 31 to 67 years) and 18 healthy volunteers (11 males, seven females, ranging in age from 32 to 66 years). Although all received respiratory coaching, two patients were unable to hold their breath and were excluded for breathholding failure, which resulted in unsolved motion artifacts even using the nonlinear image registration method. Finally, 11 patients (seven males and four females, ranging in age from 31 to 67 years) and the 18 healthy volunteers were included. There were no statistically significant between-group differences in age and sex distribution. Subsequent percutaneous renal biopsy in these 11 patients showed that six had acute tubular interstitial nephritis, two had membranous nephropathy, two had IgA nephropathy, and one had granulomatous interstitial nephritis.

RBFs were measured in the cortex and medulla of each kidney in both groups, with each group showing similar RBFs in the cortex and medulla of the left and right kidneys (Table 1).

Perfusion values in the control group ranged from 357 to $426 \mathrm{~mL} \mathrm{~min}^{-1} 100 \mathrm{~g}^{-1}$ in the cortex and from 107 to $126 \mathrm{~mL}$ $\min ^{-1} 100 \mathrm{~g}^{-1}$ in the medulla, similar to previous findings $[15,17,22]$. RBFs in patients with AKI ranged from 223 to
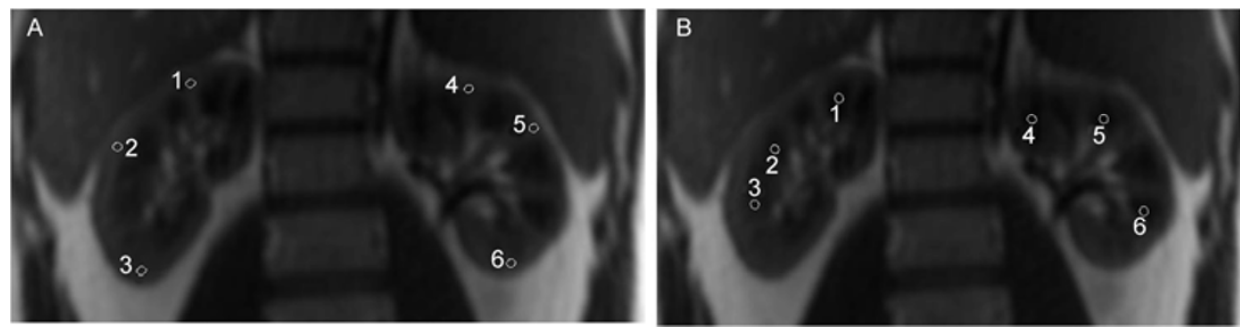

Figure 1 Regions of interests (ROIs) in the kidneys. Multiple ROIs, 50-100 $\mathrm{mm}^{2}$ in area, were drawn in the renal cortex (A) and medulla (B), with averaged values calculated as the cortical and medullary RBFs, respectively.

Table 1 RBFs $\left(\mathrm{mL} \min ^{-1} 100 \mathrm{~g}^{-1}\right)$ of the cortex and medulla in the left and right kidneys of the control and AKI groups

\begin{tabular}{lccc}
\hline & Left & Right & $P$-value \\
\hline Control & & & \\
Cortex & $396.7 \pm 17.6$ & $400.9 \pm 21.3$ & 0.78 \\
Medulla & $121.3 \pm 18.7$ & $115.9 \pm 20.2$ & 0.61 \\
Study & & & \\
Cortex & $285.8 \pm 44.1$ & $294.9 \pm 51.2$ & 0.43 \\
Medulla & $110.7 \pm 18.2$ & $101.6 \pm 15.5$ & 0.41 \\
\hline
\end{tabular}

Table 2 RBFs (mL min ${ }^{-1} 100 \mathrm{~g}^{-1}$ ) of the cortex and medulla in the control and AKI groups

\begin{tabular}{cccc}
\hline & Control & Study & $P$-value \\
\hline Cortex & $398.8 \pm 19.7$ & $291.8 \pm 47.1$ & $<0.01$ \\
Medulla & $118.0 \pm 19.8$ & $105.7 \pm 17.0$ & $<0.01$ \\
\hline
\end{tabular}

$392 \mathrm{~mL} \mathrm{~min}^{-1} 100 \mathrm{~g}^{-1}$ in the cortex and from 89 to $116 \mathrm{~mL}$ $\min ^{-1} 100 \mathrm{~g}^{-1}$ in the medulla, with both being significantly lower than in the healthy volunteers (Table 2). Functional perfusion maps of ASL showed differences between the study and control groups (Figure 2).

Serum creatinine concentrations ranged from 52 to 111 $\mu \mathrm{mol} \mathrm{L}{ }^{-1}$ in the control group and from 65.8 to $752 \mu \mathrm{mol}$ $\mathrm{L}^{-1}$ in the AKI group. In the control group, RBF in the cortex showed a significant positive correlation with serum creatinine concentration ( $r=-0.71, P=0.001)$, with no significant correlation in the medulla $(r=-0.39, P=0.24)$. In the AKI group, however, RBF in the cortex $(r=-0.31, P=0.28)$ and medulla $(r=-0.27, P=0.48)$ was not correlated with serum creatinine concentration (Figure 3 ). 

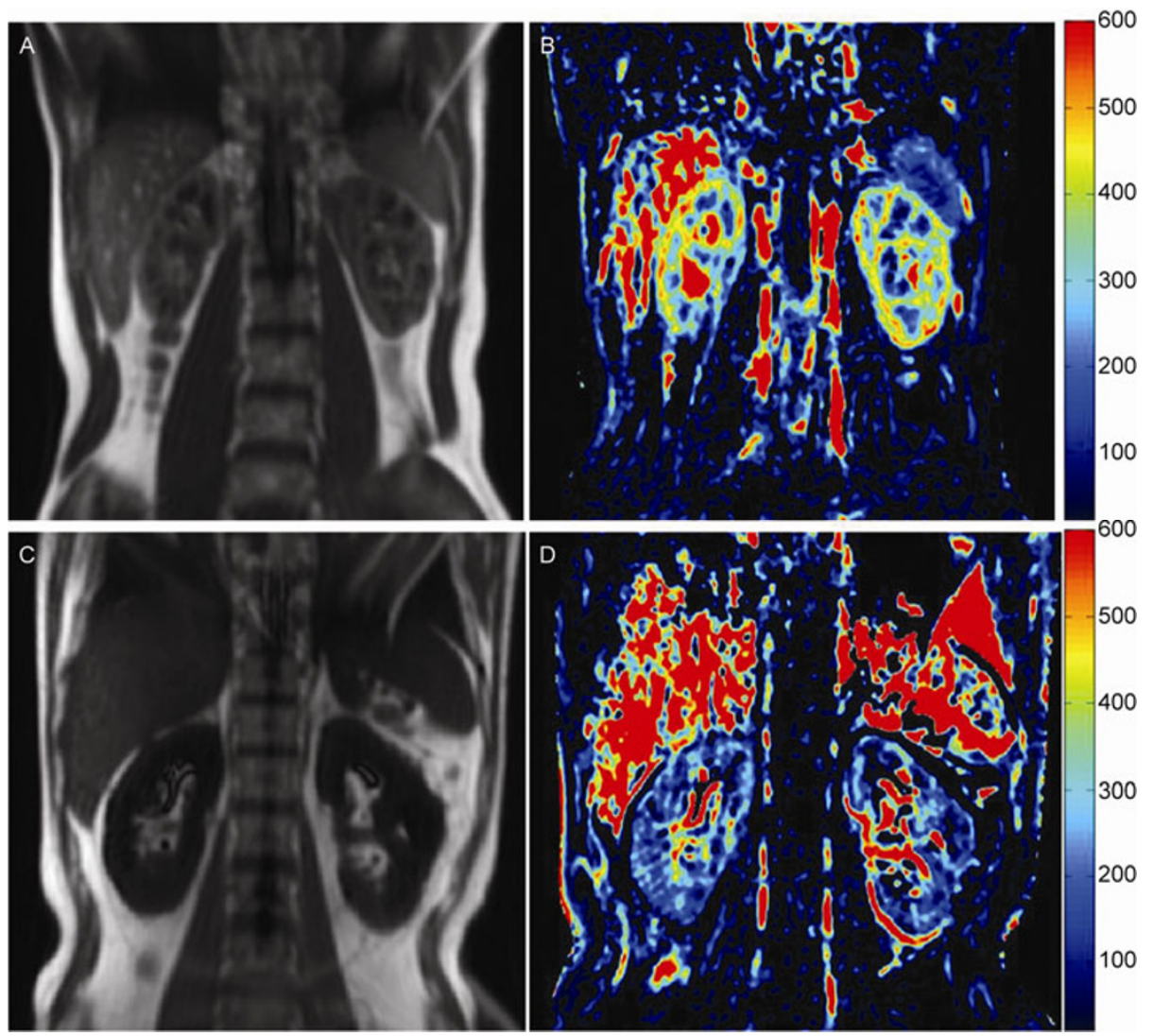

Figure 2 Oblique coronal image of ASL and perfusion map of subjects in the (A and B) and AKI (C and D) groups. A and B, Results in a 32-year-old male volunteer, showing a clear corticomedullary edge of the kidney (A), and normal renal blood flow (RBF) (cortex, $401.6 \mathrm{~mL} \mathrm{~min}^{-1} 100 \mathrm{~g}^{-1}$; medulla, $123.0 \mathrm{~mL}$ $\mathrm{min}^{-1} 100 \mathrm{~g}^{-1}$ ) (B). C and D, Results in a 48-year-old man diagnosed with tubular interstitial nephrology, showing an obscure boundary between the cortex and medulla of the kidney $(C)$, with lower RBFs in the cortex $\left(272.2 \mathrm{~mL} \mathrm{~min}^{-1} 100 \mathrm{~g}^{-1}\right)$ and medulla $\left(97.6 \mathrm{~mL} \mathrm{~min}^{-1} 100 \mathrm{~g}^{-1}\right)$ (D).

\section{Discussion}

To our knowledge, this is the first study to assess RBF using ASL MRI in patients with AKI. The results suggest that this noninvasive method, using FAIR-SSFSE sequences, yields reliable quantitative measurements of RBF in both AKI patients and healthy volunteers without administration of contrast media. Reduction in RBF in both the cortex and medulla can help diagnose AKI, especially for patients with impaired renal function.

ASL imaging, a noninvasive functional MRI technique, has been widely used to assess perfusion changes in patients with cerebral artery diseases, and has yielded promising results [23-25]. Application of ASL to the kidneys, however, is limited by respiration and magnetic susceptibility artifacts in abdominal organs. Using the nonlinear image registration method, ASL images can be acquired with acceptable quality for calculating perfusion maps of RBF. In addition, the background suppression technique was used to zero static tissue to enhance the low signal-to-noise ratio in perfusion maps, thus increasing perfusion signal [26]. ASL imaging with FAIR true FISP has been found suitable for quantitative assessment of RBF in patients with renal artery stenosis
[15]. Moreover, ASL imaging has been used in a mouse renal cell carcinoma model to characterize early changes in response to antiangiogenic therapy, with these changes predicting later therapeutic responses [14]. We found that, in healthy volunteers, mean RBF was $(398.8 \pm 19.7) \mathrm{mL} \mathrm{min}^{-1}$ $100 \mathrm{~g}^{-1}$ in the cortex and $(118 \pm 19.8) \mathrm{mL} \min ^{-1} 100 \mathrm{~g}^{-1}$ in the medulla, with no significant differences between the left and right kidneys. These results are consistent with studies showing the reliability and reproducibility of the ASL technique.

AKI refers to a clinical syndrome characterized by rapid decreases in renal function within a few hours to a few days, with accumulation of products of nitrogen metabolism such as creatinine and urea [10]. Causes of AKI vary from reduced kidney perfusion and prerenal azotemia to direct renal toxicity and postrenal obstruction, with AKI accounting for $1.9 \%$ of hospital inpatients [27]. Although increased serum creatinine and urea concentrations are the standard diagnostic laboratory criteria for AKI, they are relatively insensitive, becoming abnormal only when glomerular filtration rate decreases by more than $50 \%$. Furthermore, they do not show dynamic changes in glomerular filtration rates [28]. Although several novel biomarkers were recently re- 

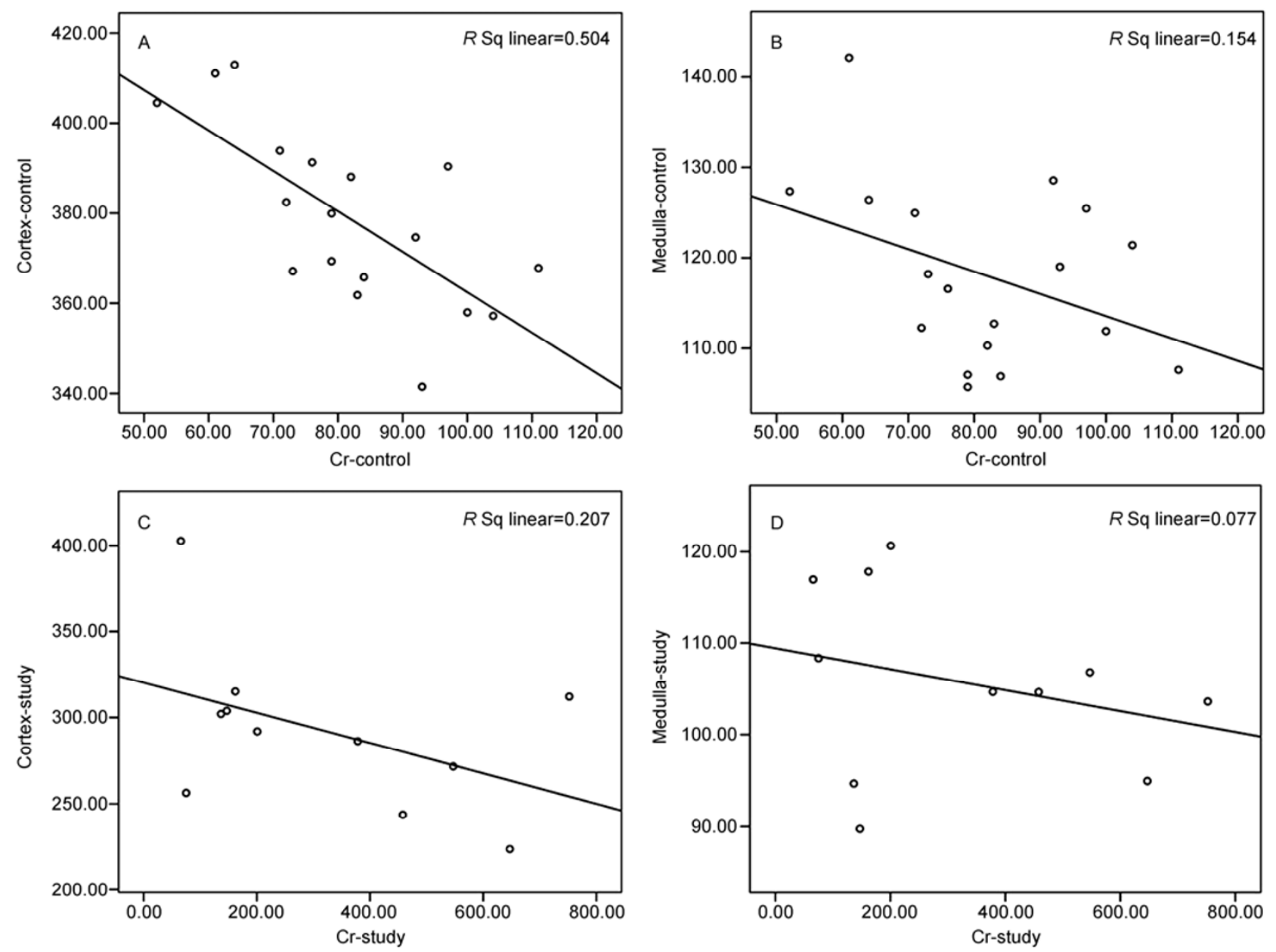

Figure 3 Correlation between serum creatinine concentration and cortical and medullary renal blood flow (RBF) in the control (A and B) and AKI (C and D) groups. A, Cortical RBF was positively correlated with serum creatinine concentration in the control group ( $r=-0.71, P=0.001)$. B-D, There were no correlations between serum creatinine concentration and medullary RBF in the control group (B) and between serum creatinine and cortical (C) and medullary (D) RBFs in the AKI group.

ported to diagnose AKI earlier and to reflect dynamic alterations to therapy, their clinical usefulness requires additional confirmation $[6,7,29]$. The dominant mechanism in the pathogenesis of AKI is a significant reduction in RBF [30]. Although multiple modalities have been used to measure $\mathrm{RBF}$, including nuclear medicine and contrast enhanced CT, impaired renal function restricts their application.

The most important advantages of ASL in quantifying $\mathrm{RBF}$ are that it is completely non-invasive method and does not require administration of contrast media. This prevents any potential risk of contrast medium induced nephrology, which is of extreme importance for patients with impaired renal function. ASL MRI has been used in patients with impaired renal allograft function, and its reproducibility and reliability have been confirmed $[11,17]$. To assess the stability of ASL, each sequence was scanned twice in each participant, with RBF determined by the average of the two perfusion values. We found that the renal perfusion examination based on our FAIR SSFSE sequence was robust, with the main perfusion signal recorded by this sequence coming from low velocity spins in capillary blood in the steady state, which stayed in the imaging slice for a relatively long period of time. RBF was significantly lower in AKI patients than in healthy volunteers, in accordance with the pathophysiological changes that occur during AKI $[5,30]$. Therefore, this noninvasive imaging modality is potentially capable of diagnosing AKI in patients with impaired renal function.

To date, AKI has been diagnosed from increases in serum creatinine concentration $[10,28]$. This relatively insensitive marker of glomerular filtration rate was found to be affected by many factors, including muscle mass, age, sex, muscle injury, use of steroids and aggressive fluid resuscitation [10]. To avoid these factors, all subjects were evaluated after a 6-h fast; moreover, their demographic and clinical characteristics were similar. The only positive correlation we observed between serum creatinine and RBF was in the cortex of the control group. The absence of a correlation between medullary RBF and serum creatinine in patients with AKI may have resulted from our inclusion of patients 
with different pathological types and the limited numbers of patients. Future studies, involving more patients and those with uniform pathology, are required.

This study had several limitations. First, the absence of a gold standard for RBF limited comparisons of quantitative measurements of ASL perfusion results. However, FAIR-ASL has shown good correlation with standard references $[15,16]$, and our results were consistent. Second, we assessed a limited number of patients with AKI and these had different pathological types. Additional studies with larger numbers of patients are therefore required. Third, we assumed that the renal $\mathrm{T} 1$ relaxation times were similar in AKI patients and healthy volunteers. However, the T1 relaxation times of the kidneys may change in patients with AKI; this should be evaluated in future studies.

In conclusion, noninvasive perfusion ASL MRI is feasible for the quantitative measurement of RBF in AKI patients without the need to administer contrast media. The significantly lower cortical, medullary and global kidney RBFs in AKI patients than in healthy volunteers suggest that this method may be useful in the diagnosis of AKI.

This work was supported by the Capital Health Research and Development Projects of Beijing (2011-4021-02).

1 Golestaneh L, Melamed M L, Hostetter T H. Uremic memory: the role of acute kidney injury in long-term outcomes. Kidney Int, 2009, 76: 813-814

2 Hsu C Y, McCulloch C E, Fan D, et al. Community-based incidence of acute renal failure. Kidney Int, 2007, 72: 208-212

3 Wald R, Quinn R R, Luo J, et al. Chronic dialysis and death among survivors of acute kidney injury requiring dialysis. JAMA, 2009, 302: 1179-1185

4 Valette X, du Cheyron D. A critical appraisal of the accuracy of the RIFLE and AKIN classifications in defining "acute kidney insufficiency" in critically ill patients. J Crit Care, 2013, 28: 116-125

5 Schrier R W, Wang W. Acute renal failure and sepsis. N Engl J Med, 2004, 351: 159-169

6 Devarajan P. Biomarkers for the early detection of acute kidney injury. Curr Opin Pediatr, 2011, 23: 194-200

7 Sirota J C, Klawitter J, Edelstein C L. Biomarkers of acute kidney injury. J Toxicol, 2011, 2011: 328120

8 Ostermann M, Philips B J, Forni L G. Clinical review: biomarkers of acute kidney injury: where are we now? Critical Care, 2012, 16: 233

9 Vanmassenhove J, Vanholder R, Nagler E, et al. Urinary and serum biomarkers for the diagnosis of acute kidney injury: an in-depth review of the literature. Nephrol Dial Transplant, 2013, 28: 254-273

10 Bellomo R, Kellum J A, Ronco C. Acute kidney injury. Lancet, 2012, 380: 756-766

11 Pedrosa I, Rafatzand K, Robson P, et al. Arterial spin labeling MR imaging for characterisation of renal masses in patients with impaired renal function: initial experience. Eur Radiol, 2012, 22: 484-492

12 Artz N S, Sadowski E A, Wentland A L, et al. Arterial spin labeling MRI for assessment of perfusion in native and transplanted kidneys. Magn Reson Imag, 2011, 29: 74-82

13 Abd E M, Kremser C, Pallwein L, et al. Changes of renal blood flow after ESWL: assessment by ASL MR imaging, contrast enhanced MR imaging, and renal resistive index. Eur J Radiol, 2010, 76: 124-128

14 Schor-Bardach R, Alsop D C, Pedrosa I, et al. Does arterial spinlabeling MR imaging-measured tumor perfusion correlate with renal cell cancer response to antiangiogenic therapy in a mouse model? Radiology, 2009, 251: 731-742

15 Fenchel M, Martirosian P, Langanke J, et al. Perfusion MR imaging with FAIR true FISP spin labeling in patients with and without renal artery stenosis: initial experience. Radiology, 2006, 238: 1013-1021

16 Wu W C, Su M Y, Chang C C, et al. Renal perfusion 3-T MR imaging: a comparative study of arterial spin labeling and dynamic contrast-enhanced techniques. Radiology, 2011, 261: 845-853

17 Artz N S, Sadowski E A, Wentland A L, et al. Reproducibility of renal perfusion MR imaging in native and transplanted kidneys using non-contrast arterial spin labeling. J Magn Reson Imag, 2011, 33: 1414-1421

18 Lanzman R S, Wittsack H J, Martirosian P, et al. Quantification of renal allograft perfusion using arterial spin labeling MRI: initial results. Eur Radiol, 2010, 20: 1485-1491

19 Roberts D A, Detre J A, Bolinger L, et al. Renal perfusion in humans: MR imaging with spin tagging of arterial water. Radiology, 1995, 196: 281-286

20 Stanisz G J, Odrobina E E, Pun J, et al. T1, T2 relaxation and magnetization transfer in tissue at 3T. Magn Reson Med, 2005, 54: 507-512

21 de Bazelaire C M, Duhamel G D, Rofsky N M, et al. MR imaging relaxation times of abdominal and pelvic tissues measured in vivo at 3.0 T: preliminary results. Radiology, 2004, 230: 652-659

22 Boss A, Martirosian P, Claussen C D, et al. Quantitative ASL muscle perfusion imaging using a FAIR-TrueFISP technique at 3.0 T. NMR Biomed, 2006, 19: 125-132

23 Bonventre J V, Yang L. Cellular pathophysiology of ischemic acute kidney injury. J Clinic Invest, 2011, 121: 4210-4221

24 Edelman R R, Siewert B, Darby D G, et al. Qualitative mapping of cerebral blood flow and functional localization with echo-planar MR imaging and signal targeting with alternating radio frequency. Radiology, 1994, 192: 513-520

25 Taoka T, Iwasaki S, Nakagawa H, et al. Distinguishing between anterior cerebral artery and middle cerebral artery perfusion by colorcoded perfusion direction mapping with arterial spin labeling. AJNR Am J Neuroradiol, 2004, 25: 248-251

26 Mani S, Pauly J, Conolly S, et al. Background suppression with multiple inversion recovery nulling: applications to projective angiography. Magn Reson Med, 1997, 37: 898-905

27 Liangos O, Wald R, O'Bell J W, et al. Epidemiology and outcomes of acute renal failure in hospitalized patients: a national survey. Clin $\mathrm{J}$ Am Soc Nephrol, 2006, 1: 43-51

28 Uchino S. Creatinine. Curr Opin Crit Care advance online publication 21 Aug 2010; doi: 10.1097/MCC.0b013e32833ea7f3

29 Goldstein S L. Acute kidney injury biomarkers: renal angina and the need for a renal troponin I. BMC Med, 2011, 9: 135

30 Prowle J R, Ishikawa K, May C N, et al. Renal blood flow during acute renal failure in man. Blood Purif, 2009, 28: 216-225

Open Access This article is distributed under the terms of the Creative Commons Attribution License which permits any use, distribution, and reproduction in any medium, provided the original author(s) and source are credited. 